\title{
Optimal Design of Noisy Transmultiplexer Systems
}

\author{
Huan Zhou ${ }^{1}$ and Lihua Xie ${ }^{2}$ \\ ${ }^{1}$ Signal Processing Group, Institute of Physics, University of Oldenburg, 26111 Oldenburg, Germany \\ ${ }^{2}$ School of Electrical and Electronic Engineering, Nanyang Technological University, Singapore 639798
}

Received 31 October 2004; Revised 26 August 2005; Accepted 19 September 2005

Recommended for Publication by Yuan-Pei Lin

\begin{abstract}
An optimal design method for noisy transmultiplexer systems is presented. For a transmultiplexer system with given transmitters and desired crosstalk attenuation, we address the problem of minimizing the reconstruction error while ensuring that the crosstalk of each band is below a prescribed level. By employing the mixed $H_{2} / H_{\infty}$ optimization, we will ensure that the system with suboptimal reconstruction error is more robust and less sensitive to the changes of input signals and channel noises. Due to the overlapping of adjacent subchannels, crosstalk between adjacent channels is expected. And the problem of crosstalk attenuation is formulated as an $H_{\infty}$ optimization problem, solved in terms of linear matrix inequalities (LMIs). The simulation examples demonstrate that the proposed design performs better than existing design methods.
\end{abstract}

Copyright ( 2006 Hindawi Publishing Corporation. All rights reserved.

\section{INTRODUCTION}

Transmultiplexers (TMUX) were studied in the early 1970's by Bellanger and Daguet [1] for telephone applications, with original intention to convert data between time division multiplexed (TDM) format and frequency-division multiplexed (FDM) format. They have been successfully utilized for multiuser communications. A multi-input multi-output (MIMO) M-band conventional TMUX system (Figure 1) with critical sampling (i.e., all interpolation factors equal to band number, also called as minimally interpolated TMUX in [2]) is well suited for simultaneous transmission of many data signals through a single channel by using the frequency-division multiplexing (FDM) technique. In traditional distortion-free $(C(z)=1$ and $r(n)=0$ in Figure 1) TMUX system, the transmitters (the left filter bank) $\left\{F_{i}(z)\right\}$ traditionally cover different uniform regions of frequency. So the signals $u_{i}(n), i=0,1, \ldots, M-1$, are packed into $M$ adjacent frequency bands (passbands of the filters) and added to obtain the composite signal $q(n)$. With the transmitters $F_{i}(z), i=0,1, \ldots, M-1$, chosen as ideal bandpass filters, we can regard $p(n)$ as a frequency-division multiplexed or FDM version of the separate signals $u_{i}(n)$, and the receivers (the right filter bank) $\left\{H_{k}(z)\right\}$ decompose this signal into $\widehat{v}_{i}(n)$, $i=0,1, \ldots, M-1$, with the decimated version of $\hat{v}_{i}(n)$ being the reconstructed signal $\hat{s}_{i}(k)$. So, the TMUX system can be seen as a complete TDM $\rightarrow$ FDM $\rightarrow$ TDM converter which is exactly the dual system of the subband filter bank system [3].
However, in the TMUX system, if the transmitters $F_{i}(z)$ are nonideal, the adjacent spectra will actually tend to overlap. Similarly, if the receivers $H_{i}(z)$ are nonideal, then the output signal of $i$ th band $\hat{s}_{i}(k)$ has contribution from the desired signal input $s_{i}(k)$ as well as input signals of other bands $s_{l}(k), l \neq i$. The leakage of signal from one band to another is known as crosstalk [4]. Such crosstalk phenomenon is basically caused by the downsampling operations and the fact that the transmitting filters $F_{i}(z)$ are not ideal, which is also one of the main problems in TMUX systems. There have been many studies in the past. Intuitively, crosstalk can be cancelled by employing nonoverlapped transmitters $F_{i}(z)$, and bandlimiting the signals $s_{i}(k)$ to $|\omega|<\sigma_{i}$ with $\sigma_{i}<\pi$, so that there is no overlap between signals of adjacent bands in the FDM format. That is, there exists a guard band between adjacent frequency bins, which ensures no crosstalk between adjacent signals, even though the filters have nonzero transition band [5]. A larger guard band implies larger permissible transition band (hence lower cost) for the receivers $H_{i}(z)$. However, the existence of guard bands results in that the channel bandwidth is not fully utilized in the transmission process. If transmitter filters $F_{i}(z)$ are ideal with very sharp cutoff and equal bandwidth $\pi / M$, the channel bandwidth is fully utilized. However, such ideal filters are of course unrealizable, and good approximations of such filters are expensive.

Although ideal filters cannot be realized in practice, the crosstalk in TMUX systems can still be cancelled by incorporating proper design of separation filters, see, for 


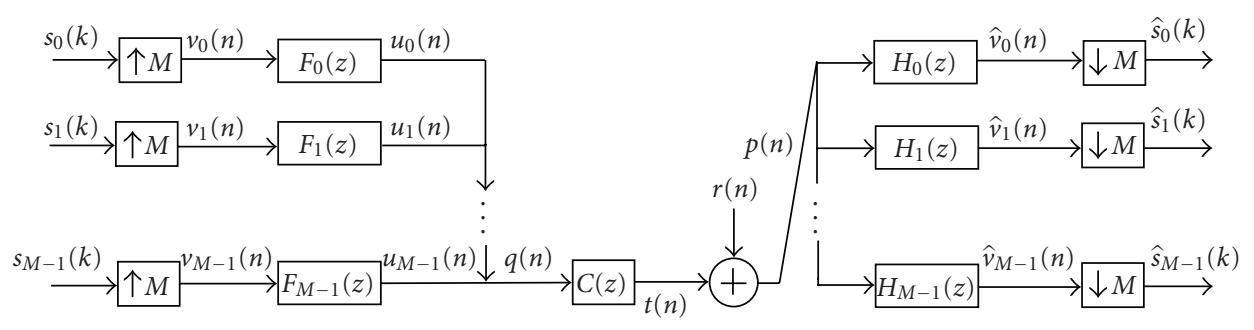

FIgURE 1: TMUX model with channel and channel noise.

example, Vetterli [6]. In this approach, crosstalk is permitted in TDM $\rightarrow$ FDM converter but is cancelled at the FDM $\rightarrow$ TDM stage. That is, even if there are no guard bands (thereby permitting crosstalk), we can eliminate the crosstalk in a manner analogous to aliasing cancellation in maximally decimated filter banks by a careful choice of transmitters and receivers. By this approach, the filters $H_{i}(z)$ and $F_{i}(z)$ are more economical than those in conventional designs. In fact, note that under certain condition perfect symbol recovery may be possible even with nonideal filters having overlapping responses, for instance, with the so-called biorthogonal filter bank [7].

For noise-free TMUX system, a lot of conventional researches have been devoted to exploit the perfect reconstruction property. As such, it has been studied from the point of view of periodically time-varying (PTV) filters in $[8,9]$, with the technique of the selection of PTV filters poles and zeros. In [10], an $\mathrm{H}_{2}$ optimization approach is used to design nonuniform-band TMUX systems, resulting in Near PR (NPR) TMUX systems. Moreover, since the quadrature mirror filter (QMF) bank and the TMUX system are dual to each other, the design of PR TMUX system can be solved by design PR QMF system, as discussed in [5].

Unfortunately, this perfect recovery is achieved under the assumption that channel effects including channel distortion and additive channel noises are negligible. For practical distorted channels, the orthogonality between bands is destroyed at the receiver, causing in most cases unacceptable performance degradation. A practical channel model is shown in Figure 1 which consists of linear FIR filter $C(z)$, with order $L<M$ (a reasonable assumption after channel equalization), and with additive noise $r(n)$, see [11]. The composite signal $p(n)$ is a distorted and noisy version of $\left\{s_{0}(k), s_{1}(k), \ldots, s_{M-1}(k)\right\}$.

For this practical noisy TMUX system, in [12], Wiener filtering approach is presented via the least-squares method to maintain the reconstruction performance, also, Chen et al. proposed a series of studies to deal with the signal reconstruction problem from the $\mathrm{H}_{2}$ optimal point of view [13-15], and recently, an MMSE approach is proposed for perfect DFT-based DMT system design [11], with the major shortcoming that the statistical properties of input and noises must be known. To improve it, $H_{\infty}$ optimization or minimax approach is developed in [16]. Moreover, in [17], a mixed $\mathrm{H}_{2} / \mathrm{H}_{\infty}$ design is developed for TMUX system with additive noise, but with much conservatism due to adopting the same
Lyapunov matrix for characterizing both the $H_{2}$ and $H_{\infty}$ performances.

In this study, we focus on a critically sampled TMUX system. It is assumed that all users are independent, that is, $s_{i}$ is independent of $s_{j}$ for $i \neq j$; and each band is allowed to have different delays $d_{i}$ for constructing its input. Both the transmitters and receivers are assumed to be FIR filters and channel noise $r(n)$ is a white noise [11]. We address the problem of minimizing the reconstruction error while ensuring that the crosstalk is below certain level in the presence of channel noise. We will first design optimal and robust receivers to reconstruct the input signals with the optimal reconstruction error in the noisy channel. For the crosstalk optimization problem, some $H_{\infty}$ constraints are added to ensure the TMUX system within desired crosstalk attenuation levels. Our solution is given in terms of linear matrix inequalities (LMIs) which can be solved easily by convex optimization [18]. As illustrated later, compared with the existing TMUX design method via LMI technique [17], the proposed method embodies two obvious advantages. First, when the reconstruction performance is concerned, the proposed mixed $\mathrm{H}_{2} / \mathrm{H}_{\infty}$ optimization method provides less conservative results. Second, a multiobjective TMUX system issue has been explored in this study, in particular, the issue on both optimal reconstruction performance and the crosstalk attenuation is novelly formulated and solved via LMI technique.

\section{2. $\mathrm{H}_{2}$ OPTIMIZATION ON RECONSTRUCTION ERROR}

In this section, we will establish the state-space model of the noisy TMUX system first, then formulate its $\mathrm{H}_{2}$ optimization by LMIs.

Remark 1. In a practical TMUX system, most TMUX systems apply an FIR equalizer in order to shorten the effective length of the physical channel impulse response, modeled as an FIR filter $C(z)$ with order $L$ (usually, the order $L$ of $C(z)$ is smaller than the interpolation factor $M$ [2], which is called as the LS shortening [19]), and may be multichannel case $C_{i}(z)(i=0,1, \ldots, M-1)$ in some TMUX system applications. For the convenience of further discussion, throughout the paper, we will combine each transmitting filter $F_{i}(z)$ with subchannel $C_{i}(z)$ together, and describe the $C_{i}(z) F_{i}(z)$ as new transmitting filter $F_{i}(z)$, without specific explanation. 


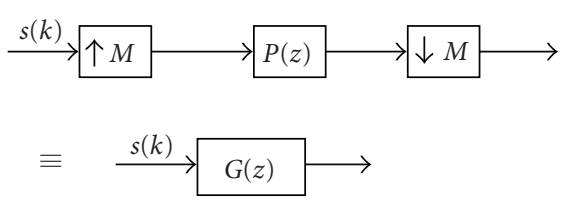

FIGURE 2: The polyphase identity.

\subsection{State-space description of TMUX systems}

Note that even though the decimator and expander are timevarying building blocks, the cascaded system shown in Figure 2 is in fact time invariant from an input and output point of view, which is the so-called property of polyphase identity [5]. That is,

$$
\left.\left[S\left(z^{M}\right) P(z)\right]\right|_{\downarrow_{M}}=S(z)\left[\left.P(z)\right|_{{ } M}\right]=S(z) G(z),
$$

where $G(z)$ is the 0 th polyphase component of $P(z)$ and $S(z)$ is the $z$-transform of the input $s(k)$.

As shown in Figure 1, by the polyphase identity property, we know that the TMUX system is an $M$-input $M$-output LTI systems. To facilitate later analysis, here we assume the maximum channel delays as $d$, the maximum length of $M$ transmitting filters as $l_{f}$ and $l_{h}$ for $M$ receiving filters. Now we analyze the system via a state-space approach.

Let $\bar{v}_{j}(k), \bar{u}_{j}(k), \bar{r}(k), \bar{p}(k)$, and $\overline{\hat{v}}_{j}(k)(j=0,1, \ldots, M-$ 1) be the vector representations of the $j$ th $M$-block of the signals $v_{j}(n), u_{j}(n), r(n), p(n)$, and $\hat{v}_{j}(n)$, respectively. For example,

$$
\begin{gathered}
\bar{v}_{j}(k)=\left[v_{j}(n), v_{j}(n+1), \ldots, v_{j}(n+M-1)\right]^{T} \in \mathcal{R}^{M}, \\
n=k M .
\end{gathered}
$$

It is clear that

$$
\bar{v}_{j}(k)=\left[\begin{array}{llll}
1 & 0 & \cdots & 0
\end{array}\right]^{T} s_{j}(k)=\alpha s_{j}(k),
$$

where $\alpha=\left[\begin{array}{llll}1 & 0 & \cdots & 0^{T}\end{array}\right]$. The transmitter $F_{j}$ is assumed to have the following state-space realization:

$$
\begin{gathered}
x_{j}^{f}(n+1)=A_{f, j} x_{j}^{f}(n)+B_{f, j} v_{j}(n), \\
u_{j}(n)=C_{f, j} x_{j}^{f}(n)+D_{f, j} v_{j}(n) .
\end{gathered}
$$

By lifting the input and output of the filter $F_{j}$ ( $M$-blocking) and considering (3), we get

$$
\begin{gathered}
\bar{x}_{j}^{f}(k+1)=\bar{A}_{f, j} \bar{x}_{j}^{f}(k)+\bar{B}_{f, j} s_{j}(k), \\
\bar{u}_{j}(k)=\bar{C}_{f, j} \bar{x}_{j}^{f}(k)+\bar{D}_{f, j} s_{j}(k),
\end{gathered}
$$

where

$$
\begin{array}{cc}
\bar{A}_{f, j}=\left[A_{f, j}^{M}\right]_{l_{f} \times l_{f}}, & \bar{B}_{f, j}=\left[A_{f, j}^{M-1} B_{f, j}\right]_{l_{f} \times 1}, \\
\bar{C}_{f, j}=\left[\begin{array}{c}
C_{f, j} \\
C_{f, j} A_{f, j} \\
\vdots \\
C_{f, j} A_{f, j}^{M-1}
\end{array}\right]_{M \times l_{f}}, \quad \bar{D}_{f, j}=\left[\begin{array}{c}
D_{f, j} \\
C_{f, j} B_{f, j} \\
C_{f, j} A_{f, j} B_{f, j} \\
\vdots \\
C_{f, j} A_{f, j}^{M-2} B_{f, j}
\end{array}\right]_{M \times 1} .
\end{array}
$$

Then block all inputs $s_{j}(k)$ and outputs of synthesis filter bank $\bar{u}_{j}(k)$, that is,

$$
\begin{aligned}
\bar{s}(k) & =\left[s_{0}(k), s_{1}(k), \ldots, s_{M-1}(k)\right]^{T} \in \mathcal{R}^{M}, \\
\overline{\bar{u}}(k) & =\left[\bar{u}_{0}^{T}(k), \bar{u}_{1}^{T}(k), \ldots, \bar{u}_{M-1}^{T}(k)\right]^{T} \in \mathcal{R}^{M^{2}} .
\end{aligned}
$$

A state-space realization of the model of the transmitter system from $\left\{s_{0}(k), \ldots, s_{M-1}(k)\right\} \rightarrow\left\{\bar{u}_{0}(k), \ldots, \bar{u}_{M-1}(k)\right\}$ can be obtained as

$$
\begin{gathered}
X^{f}(k+1)=\mathcal{A}^{f} \mathcal{X}^{f}(k)+\mathscr{B}^{f} \bar{s}(k), \\
\bar{u}(k)=\mathcal{C}^{f} \mathcal{X}^{f}(k)+\mathscr{D}^{f} \bar{s}(k),
\end{gathered}
$$

where

$$
\begin{aligned}
\chi^{f}(k) & =\left[\bar{x}_{0}^{f}(k), \bar{x}_{1}^{f}(k), \ldots, \bar{x}_{M-1}^{f}(k)\right]^{T}, \\
\mathcal{A}^{f} & =\operatorname{diag}\left\{\bar{A}_{f, 0}, \ldots, \bar{A}_{f, M-1}\right\}, \\
\mathscr{B}^{f} & =\operatorname{diag}\left\{\bar{B}_{f, 0}, \ldots, \bar{B}_{f, M-1}\right\}, \\
\mathcal{C}^{f} & =\operatorname{diag}\left\{\bar{C}_{f, 0}, \ldots, \bar{C}_{f, M-1}\right\}, \\
\mathscr{D}^{f} & =\operatorname{diag}\left\{\bar{D}_{f, 0}, \ldots, \bar{D}_{f, M-1}\right\}
\end{aligned}
$$

with $\mathscr{A}^{f} \in \mathcal{R}^{M l_{f} \times M l_{f}}, \mathscr{B}^{f} \in \mathcal{R}^{M l_{f} \times M}, \mathcal{C}^{f} \in \mathcal{R}^{M^{2} \times M l_{f}}$, and $\mathscr{D}^{f} \in \mathcal{R}^{M^{2} \times M}$. So the channel input $q(n)$ is followed by

$$
\left.\bar{q}(k)=\beta \overline{\bar{u}}_{(}\right),
$$

where $\beta=\left[I_{M}, I_{M}, \ldots, I_{M}\right] \in \mathcal{R}^{M \times M^{2}}$. Together with blocked channel noise $\bar{r}(k)$, which is assumed as a white Gaussian noise with variance $\sigma_{r}^{2}$ and independent of the input signal $\bar{s}(k)$, the input of receivers is $\bar{p}(k)=\bar{q}(k)+\bar{r}(k)$.

Similarly, for the receivers, let the state-space realization of the receiver $H_{j}(z)$ be given by

$$
\begin{gathered}
x_{j}^{h}(n+1)=A_{h, j} x_{j}^{h}(n)+B_{h, j} p(n), \\
\hat{v}_{j}(n)=C_{h, j} x_{j}^{h}(n)+D_{h, j} p(n) .
\end{gathered}
$$

By applying the lifting technique and taking into account the fact that the output of the $j$ th band is

$$
\widehat{s}_{j}(k)=\left[\begin{array}{llll}
1 & 0 & \cdots & 0
\end{array}\right] \overline{\hat{v}}_{j}(k)=\alpha^{T} \overline{\hat{v}}_{j}(k),
$$

where $\overline{\hat{v}}_{j}(k)$ is the lifted output of $\hat{v}_{j}(k)$, considering (13), we have

$$
\begin{gathered}
\bar{x}_{j}^{h}(k+1)=\bar{A}_{h, j} \bar{x}_{k}^{h}(k)+\bar{B}_{h, j} \bar{p}(k), \\
\hat{s}_{j}(k)=C_{h, j} \bar{x}_{k}^{h}(k)+\bar{D}_{h, j} \bar{p}(k),
\end{gathered}
$$

where

$$
\begin{gathered}
\bar{A}_{h, j}=A_{h, j}^{M} \in \mathcal{R}^{l_{h} \times l_{h}}, \\
\bar{B}_{h, j}=\left[A_{h, j}^{M-1} B_{h, j}, A_{h, j}^{M-2} B_{h, j}, \ldots, A_{h, j} B_{h, j}, B_{h, j}\right] \in \mathcal{R}^{l_{h} \times M}, \\
\bar{D}_{h, j}=\left[\begin{array}{lllll}
D_{h, j} & 0 & 0 & \cdots & 0
\end{array}\right] \in \mathcal{R}^{1 \times M} .
\end{gathered}
$$


Denote

$$
\overline{\widehat{s}}(k)=\left[\begin{array}{llll}
\hat{s}_{0}(k) & \hat{s}_{1}(k) & \cdots & \hat{s}_{M-1}(k)
\end{array}\right]^{T} .
$$

Then the receiver system can be represented by the following blocked state-space equations:

$$
\begin{gathered}
X^{h}(k+1)=\mathscr{A}^{h} X^{h}(k)+\mathscr{B}^{h} \bar{p}(k), \\
\overline{\hat{s}}(k)=\mathcal{C}^{h} X^{h}(k)+D^{h} \bar{p}(k),
\end{gathered}
$$

where

$$
\begin{aligned}
& X^{h}(k)=\left[\bar{x}_{0}^{h}(k), \bar{x}_{1}^{h}(k), \ldots, \bar{x}_{M-1}^{h}(k)\right]^{T}, \\
& \mathscr{A}^{h}=\operatorname{diag}\left\{\bar{A}_{h, 0}, \ldots, \bar{A}_{h, M-1}\right\}, \quad \mathscr{B}^{h}=\left[\bar{B}_{h, 0}^{T}, \ldots, \bar{B}_{h, M-1}^{T}\right]^{T}, \\
& \mathcal{C}^{h}=\operatorname{diag}\left\{C_{h, 0}, \ldots, C_{h, M-1}\right\}, \quad \mathbb{D}^{h}=\left[\bar{D}_{h, 0}^{T}, \ldots, \bar{D}_{h, M-1}^{T}\right]^{T}
\end{aligned}
$$

with $\mathscr{A}^{h} \in \mathcal{R}^{M l_{h} \times M l_{h}}, \mathscr{B}^{h} \in \mathcal{R}^{M l_{h} \times M}, \mathcal{C}^{h} \in \mathcal{R}^{M \times M l_{h}}$, and $\mathscr{D}^{h} \in \mathcal{R}^{M \times M^{2}}$.

Let $d_{j}$ be the allowable delay in reconstructing the signal $s_{j}(k)$, with $d=\max \left(d_{0}, d_{1}, \ldots, d_{M-1}\right)$. A state-space realization of the $d_{j}$-shift $\delta\left(n-d_{j}\right)$ is written as

$$
x_{j}^{d}(k+1)=A_{j}^{d} x_{j}^{d}(k)+B_{j}^{d} s_{j}(k), \quad s_{j}^{d}(k)=C_{j}^{d} x_{j}^{d}(k),
$$

where

$$
\begin{gathered}
A^{d}=\left[\begin{array}{cc}
0 & I_{d-1} \\
0 & 0
\end{array}\right] \in \mathcal{R}^{d \times d}, \quad B^{d}=\left[\begin{array}{c}
0 \\
\vdots \\
0 \\
1
\end{array}\right] \in \mathcal{R}^{d \times 1}, \\
C_{j}^{d}=[\overbrace{0, \ldots, 0}^{d-d_{j}}, 1 \overbrace{0, \ldots, 0}^{d_{j}-1}] \in \mathcal{R}^{1 \times d} .
\end{gathered}
$$

By combining the delay models of all the $M$-bands together, we have

$$
\begin{gathered}
X^{d}(k+1)=\mathcal{A}^{d} X^{d}(k)+\mathscr{B}^{d} \bar{s}(k), \\
\bar{s}^{d}(k)=\mathcal{C}^{d} X^{d}(k),
\end{gathered}
$$

where $\mathcal{X}^{d}(k)=\left[\bar{x}_{0}^{d}(k), \bar{x}_{1}^{d}(k), \ldots, \bar{x}_{M-1}^{d}(k)\right]^{T}$, and

$$
\begin{aligned}
& \mathcal{A}^{d}=\operatorname{diag}\left\{A_{0}^{d}, \ldots, A_{M-1}^{d}\right\} \in \mathcal{R}^{M d \times M d}, \\
& \mathcal{B}^{d}=\operatorname{diag}\left\{B_{0}^{d}, \ldots, B_{M-1}^{d}\right\} \in \mathcal{R}^{M d \times M}, \\
& \mathcal{C}^{d}=\operatorname{diag}\left\{C_{0}^{d}, \ldots, C_{M-1}^{d}\right\} \in \mathcal{R}^{M \times M d} .
\end{aligned}
$$

Following from (8) and (21), for a TMUX system $\&$ with FIR transmitters and receivers, its IO relation between the TMUX inputs and reconstruction error is given by

$$
(\mathcal{E}): \begin{gathered}
X(k+1)=\mathcal{A} X(k)+\mathscr{B} \widetilde{s}(k), \\
\bar{e}(k)=\mathcal{C} \mathcal{X}(k)+\mathscr{D} \widetilde{s}(k),
\end{gathered}
$$

where $\bar{e}(k)=\overline{\widehat{s}}(k)-\bar{s}^{d}(k)$, the state vector

$$
\begin{aligned}
X(k) & =\left[\begin{array}{lll}
X^{d T} & X^{f T}(k) & X^{h T}(k)
\end{array}\right]^{T}, \\
\widetilde{s}(k) & =\left[\begin{array}{ll}
\bar{s}^{T}(k) & \bar{r}^{T}(k)
\end{array}\right]^{T},
\end{aligned}
$$

and

$$
\begin{aligned}
& \mathcal{A}=\left[\begin{array}{ccc}
\mathscr{A}^{d} & 0 & 0 \\
0 & \mathcal{A}^{f} & 0 \\
0 & \mathscr{B}^{h} \beta \mathcal{C}^{f} & \mathscr{A}^{h}
\end{array}\right], \quad \mathscr{B}=\left[\begin{array}{cc}
\mathscr{B}^{d} & 0 \\
\mathcal{B}^{f} & 0 \\
\mathscr{B}^{h} \beta \mathscr{D}^{f} & \mathscr{B}^{h}
\end{array}\right], \\
& \mathcal{C}=\left[\begin{array}{lll}
-\mathcal{C}^{d} & \mathscr{D}^{h} \beta \mathcal{C}^{f} & \mathcal{C}^{h}
\end{array}\right], \quad \mathscr{D}=\left[\begin{array}{lll}
\mathscr{D}^{h} \beta \mathscr{D}^{f} & \mathscr{D}^{h}
\end{array}\right]
\end{aligned}
$$

with $\mathcal{A} \in \mathcal{R}^{M\left(d+l_{f}+l_{h}\right) \times M\left(d+l_{f}+l_{h}\right)}, \mathscr{B} \in \mathcal{R}^{M\left(d+l_{f}+l_{h}\right) \times 2 M}, \mathcal{C} \in$ $\mathcal{R}^{M \times M\left(d+l_{f}+l_{h}\right)}$, and $\mathscr{D} \in \mathcal{R}^{M \times 2 M}$.

\section{2. $\mathrm{H}_{2}$ optimization on reconstruction error}

Given the transmitter system (22) and allowable system delays, the receiver system in the form of (14) (for $j=0,1, \ldots$, $M-1)$ can be designed such that the error system $\mathcal{E}$ in the form of (23) is stable and its $\mathrm{H}_{2}$ norm is minimized. by

Formally, as is well known, the $H_{2}$ norm of $\mathscr{E}$ is described

$$
\|\mathscr{E}\|_{2}^{2}=\operatorname{trace}\left(\mathscr{B}^{T} Q \mathscr{B}\right),
$$

where $Q$ is the observation grammian of the pair $(\mathcal{A}, \mathcal{C})$, which is the unique solution of the Lyapunov equation

$$
\mathcal{A}^{T} Q \mathcal{A}-Q+\mathcal{C}^{T} \mathcal{C}=0 .
$$

Having recast the problem as above, we now use the LMI approach [20] to solve it.

Theorem 1. The optimal receiver system for the noisy TMUX system can be solved by the optimization:

$$
\min _{S, Q, \mathcal{C}^{h}, \mathscr{D}^{h}}\|\mathcal{E}\|_{2}^{2}=\min _{S, Q, \mathcal{C}^{h}, \mathscr{D}^{h}} \operatorname{trace}(S)
$$

subject to

$$
\begin{aligned}
L_{1} & =\left[\begin{array}{ccc}
-S & \mathcal{B}^{T} Q & D^{T} \\
Q \mathscr{B} & -Q & 0 \\
\mathcal{D} & 0 & -I
\end{array}\right]<0, \\
L_{2} & =\left[\begin{array}{ccc}
-Q & \mathcal{A}^{T} Q & \mathcal{C}^{T} \\
Q \mathcal{A} & -Q & 0 \\
\mathcal{C} & 0 & -I
\end{array}\right]<0,
\end{aligned}
$$

where $\mathcal{A}, \mathcal{B}, \mathcal{C}$, and $\mathbb{D}$ are defined in (25), and $S=S^{T}$ and $Q=Q^{T}$.

The proof of the theorem readily follows from the way the problem is formulated and applying the Schur complements to (26) and (27).

Remark 2. It can be observed that (29) are linear in $Q, S$, and receiver parameters $C_{h, j}, D_{h, j}$ (for $j=0,1, \ldots, M-1$ ), which are involved in $\mathcal{C}^{h}$ and $\mathbb{D}^{h}$. Thus, the optimization in the theorem is convex and the powerful LMI toolbox [18] can be employed to obtain the $\mathrm{H}_{2}$ optimal receiver system efficiently. 


\section{MIXED $H_{2} / H_{\infty}$ OPTIMIZATION ON RECONSTRUCTION ERROR}

It is well known that one of the major drawbacks of $\mathrm{H}_{2}$ optimization is that the statistical properties (or the models) of the input signals and channel noises must be well known beforehand. To deal with general noisy TMUX system, we consider a worst-case reconstruction error, such performance can be very effectively described using $H_{\infty}$ related criteria.

To optimize the average $\left(H_{2}\right)$ reconstruction performance while ensuring a certain level of the worst-case error energy over all possible inputs and channel noises, the mixed $\mathrm{H}_{2} / \mathrm{H}_{\infty}$ optimization is to be sought.

If the error system (23) is stable, its $H_{\infty}$ norm is defined as

$$
\|\mathscr{E}\|_{\infty}=\sup _{\|\tilde{s}\|_{2} \neq 0} \frac{\|\bar{e}\|_{2}}{\|\tilde{s}\|_{2}}
$$

Moreover, its value is bounded by a prescribed scalar $\gamma$ if and only if the following inequality holds:

$$
\left[\begin{array}{cccc}
-P & \mathcal{A}^{T} P & 0 & \mathcal{C}^{T} \\
P \mathcal{A} & -P & P \mathcal{B} & 0 \\
0 & \mathcal{B}^{T} P & -\gamma I & \mathscr{D}^{T} \\
\mathcal{C} & 0 & \mathcal{D} & -\gamma I
\end{array}\right]<0 .
$$

Proof. Equation (31) can be easily derived by applying the Schur complements and the well-known bounded real lemma. lows.

Then the mixed $\mathrm{H}_{2} / \mathrm{H}_{\infty}$ optimization can be solved as fol-

Theorem 2. Give a scalar $\gamma>0$, the mixed $H_{2}$ and $H_{\infty}$ reconstruction problem is solvable if and only if the $H_{\infty}$ reconstruction problem is solvable. In this situation, the optimal mixed $\mathrm{H}_{2}$ and $\mathrm{H}_{\infty}$ receivers can be obtained by the following convex optimization:

$$
\|\mathcal{E}\|_{2}^{2}=\min _{S, Q, P, \mathbb{C}^{h}, \mathbb{D}^{h}} \operatorname{trace}(S)
$$

subject to LMIs (29), and (31), with $S=S^{T}, Q=Q^{T}$, and $P=P^{T}$.

Remark 3. Note that in [17], a mixed $H_{2} / H_{\infty}$ approach is proposed for the design of IIR receivers for a noisy TMUX system. The approach of [17] is generally conservative due to the fact that the same Lyapunov matrix is adopted for both the $H_{2}$ and $H_{\infty}$ performances. That is, only an upper bound on the $\mathrm{H}_{2}$ performance (suboptimal mixed $\mathrm{H}_{2} / \mathrm{H}_{\infty}$ receivers) is achieved. In the above, we proposed a mixed $H_{2} / H_{\infty}$ design for TMUX systems via a convex optimization which allows different Lyapunov matrices $Q$ and $P$ for the $H_{2}$ and $H_{\infty}$ performances. The result of Theorem 2 is necessary and sufficient. That is, it will lead to the optimal solution rather than a suboptimal solution.

\section{4. $H_{\infty}$ OPTIMIZATION ON CROSSTALK ATTENUATION}

In this section, we will deal with the crosstalk problem by an $H_{\infty}$ optimization approach. In general, there are two reasons for the study of crosstalk attenuation by $H_{\infty}$ approach.

First, as stated before, one problem often encountered in a TMUX system is crosstalk, for example, the crosstalk between multiple services transmitting through the same telephone cable is the primary limitation to digital subscriber line services [21]. Usually, special requirement on system crosstalk performance is imposed, for example, in the British telecommunication specifications, for a 60-channel TMUX, at least $60 \mathrm{~dB}$ interchannel crosstalk attenuation is required [8], which is a less strict requirement than crosstalk cancellation, means less cost for implementation.

The second is, in TMUX system, there are many factors resulting in modeling uncertainty, which, in most cases, may destroy the perfect crosstalk cancellation property and cause unacceptable performance degradation [12]. So, with $H_{\infty}$ optimization, crosstalk can be controlled even from the worst-case point of view.

\subsection{Problem formulation}

As stated before, the leakage from one band to another is known as the crosstalk which is the effect of other band inputs $s_{l}(k), l \neq i$, on the $i$ th band output $\widehat{s}_{i}(k), i=0,1, \ldots, M-1$.

Apply the polyphase identity to the TMUX system in Figure 2 and define $P_{i j}(z)=H_{i}(z) C(z) F_{j}(z)$ and $G_{i j}(z)$ the 0th polyphase component of $P_{i j}(z)$. Then, the output of the $i$ th band is given as

$$
\hat{S}_{i}(z)=G_{i i}(z) S_{i}(z)+\sum_{j=0, j \neq i}^{M-1} G_{i j}(z) S_{j}(z)=\widehat{S}_{i i}(z)+\widehat{S}_{c, i}(z)
$$

where $S_{i}(z)$ is the $z$-transform of $s_{i}(k)$ and $\widehat{S}_{c, i}(z)$ is due to the inputs of other bands and is termed as crosstalk in the $i$ th band.

In general, the crosstalk in the $i$ th band is composed of $(M-1)$ leakages from $(M-1)$ input $s_{j}, j=0, \ldots, i-1, i+$ $1, \ldots, M-1$. However, this can be simplified considerably if we assume that crosstalk only appears between adjacent channels [3], that is, for a TMUX system, $H_{i}(z)$ and $F_{i}(z)$ have the same frequency support domain and $H_{i}(z) H_{j}(z) \approx$ 0 for $|i-j|>1$ (nonadjacent filters practically do not overlap). This means that the expression of the $i$ th band crosstalk distortion $\hat{s}_{c, i}(n)$ for $1 \leq i \leq M-2$ contains two significant terms as $F_{i}$ practically overlaps only with $F_{i-1}$ and $F_{i+1}$. For $i=0$ or $i=M-1$ it contains only one significant term as $F_{0}$ overlaps only with $F_{1}$ and $F_{M-1}$ with $F_{M-2}$.

We will now derive a state-space representation for each crosstalk by a lifting approach, it is clear that such representation is a special case of (23), by ignoring the delays and only considering $s_{i-1}(k)$, and $s_{i+1}(k)$ being sources of the $i$ th crosstalk output.

Let $\mathcal{F}_{i}$ denote the mapping $\left(s_{i-1}, s_{i+1}\right) \mapsto \widehat{s}_{c, i}$ in the system of Figure 3. 


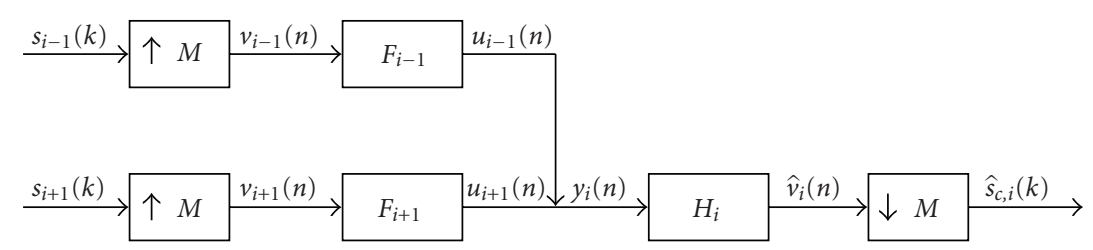

FIgURE 3: Composition of the ith crosstalk.

Denote

$$
s_{c, i}(k)=\left[\begin{array}{c}
s_{i-1}(k) \\
s_{i+1}(k)
\end{array}\right] .
$$

Following the similar derivation as above, the crosstalk of the $i$ th band is given by

$$
\begin{gathered}
\mathcal{X}_{c, i}(k+1)=\mathcal{A}_{c, i} \mathcal{X}_{c, i}(k)+\mathcal{B}_{c, i} s_{c, i}(k), \\
\left(\mathcal{E}_{c, i}\right): \hat{s}_{c, i}(k)=\mathcal{C}_{c, i} \mathcal{X}_{c, i}(k)+\mathcal{D}_{c, i} s_{c, i}(k), \quad i=1, \ldots, M-2,
\end{gathered}
$$

where the state vector $\mathcal{X}_{c, i}(k)=\left[\begin{array}{lll}\bar{x}_{i-1}^{f T}(k) & \bar{x}_{i+1}^{f T}(k) & \bar{x}_{i}^{h T}(k)\end{array}\right]^{T}$, and

$$
\begin{aligned}
& \mathcal{A}_{c, i}=\left[\begin{array}{ccc}
\bar{A}_{f, i-1} & 0 & 0 \\
0 & \bar{A}_{f, i+1} & 0 \\
\bar{B}_{h, i} \bar{C}_{f, i-1} & \bar{B}_{h, i} \bar{C}_{f, i+1} & \bar{A}_{h, i}
\end{array}\right], \\
& \mathscr{B}_{c, i}=\left[\begin{array}{cc}
\bar{B}_{f, i-1} & 0 \\
0 & \bar{B}_{f, i+1} \\
\bar{B}_{h, i} \bar{D}_{f, i-1} & \bar{B}_{h, i} \bar{D}_{f, i+1}
\end{array}\right], \\
& \mathcal{C}_{c, i}=\left[\begin{array}{lll}
D_{h, i} C_{f, i-1} & D_{h, i} C_{f, i+1} & C_{h, i}
\end{array}\right], \\
& \mathscr{D}_{c, i}=D_{h, i}\left[\begin{array}{ll}
D_{f, i-1} & D_{f, i+1}
\end{array}\right]
\end{aligned}
$$

with $\mathcal{A}_{c, i} \in \mathcal{R}^{\left(2 l_{f}+l_{h}\right) \times\left(2 l_{f}+l_{h}\right)}, \mathcal{B}_{c, i} \in \mathcal{R}^{\left(2 l_{f}+l_{h}\right) \times 2}, \mathcal{C}_{c, i} \in$ $\mathcal{R}^{1 \times\left(2 l_{f}+l_{h}\right)}$, and $\mathcal{D}_{c, i} \in \mathcal{R}^{1 \times 2}$.

The state-space realizations for the crosstalks in 0th and $(M-1)$ th bands are

$$
\begin{gathered}
\left(\mathcal{E}_{c, 0}\right): \mathcal{X}_{0}(k+1)=\mathcal{A}_{c, 0} \mathcal{X}_{0}(k)+\mathcal{B}_{c, 0} s_{1}(k), \\
\widehat{s}_{c, 0}(k)=\mathcal{C}_{c, 0} \mathcal{X}_{0}(k)+\mathcal{D}_{c, 0} s_{1}(k) ; \\
\left(\mathcal{E}_{c, M-1}\right): \mathcal{X}_{M-1}(k+1)=\mathcal{A}_{c, M-1} \mathcal{X}_{M-1}(k)+\mathcal{B}_{c, M-1} s_{M-2}(k), \\
\widehat{s}_{c, M-1}(k)=\mathcal{C}_{c, M-1} \mathcal{X}_{M-1}(k)+\mathcal{D}_{c, M-1} s_{M-2}(k),
\end{gathered}
$$

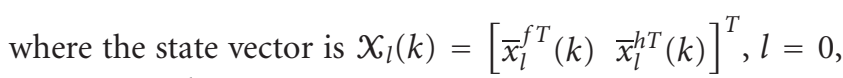
or $M-1$ and

$$
\begin{aligned}
& \mathcal{A}_{c, l}=\left[\begin{array}{cc}
\bar{A}_{f, l} & 0 \\
\bar{B}_{h, l} \bar{C}_{f, l} & \bar{A}_{h, l}
\end{array}\right] \in \mathcal{R}^{\left(l_{f}+l_{h}\right) \times\left(l_{f}+l_{h}\right)}, \\
& \mathcal{B}_{c, l}=\left[\begin{array}{c}
\bar{B}_{f, l} \\
\bar{B}_{h, l} \bar{D}_{f, l}
\end{array}\right] \in \mathcal{R}^{\left(l_{f}+l_{h}\right) \times 1}, \\
& \mathcal{C}_{c, l}=\left[\begin{array}{ll}
D_{h, l} C_{f, l} & C_{h, l}
\end{array}\right] \in \mathcal{R}^{1 \times\left(l_{f}+l_{h}\right)}, \quad \mathcal{D}_{c, l}=D_{h, l} D_{f, l} \in \mathcal{R} .
\end{aligned}
$$

\section{2. $H_{\infty}$ crosstalk attenuation}

In this subsection, we will formulate the crosstalk attenuation problem as an $H_{\infty}$ performance problem.

Assume that each input $s_{i}$ (for $i=0,1, \ldots, M-1$ ) is energy bounded, that is, $\sum_{k=0}^{\infty} s_{i}^{2}(k)<\infty$. We define the following signal-to-crosstalk ratio (SCR) to measure the crosstalk attenuation. For the given transmitters $F_{i}(z), i=0,1, \ldots, M-1$, and a desirable SCR $\rho_{i}$, design the receivers $H_{i}(z), i=0$, $1, \ldots, M-1$, such that for each $i$,

$$
\mathrm{SCR}_{i}=10 \log _{10} \frac{\sum_{k=0}^{\infty} s_{c, i}^{2}(k)}{\sum_{k=0}^{\infty} \hat{s}_{c, i}^{2}(k)}=10 \log _{10} \frac{\left\|s_{c, i}\right\|_{2}^{2}}{\left\|\hat{s}_{c, i}\right\|_{2}^{2}} \geq \rho_{i},
$$

where $s_{c, i}(k)$ is defined in (34) for $i=1,2, \ldots, M-2$, and $s_{c, 0}(k)=s_{1}(k)$ and $s_{c, M-1}(k)=s_{M-2}(k)$. Note that $\mathrm{SCR}_{i}$ as defined above is in fact to measure the ratio of the input energy and output energy of $\mathcal{E}_{c, i}$. Let $\gamma_{i}=10^{-\rho_{i} / 10}$. It is easy to know that (39) is equivalent to

$$
\left\|\varepsilon_{c, i}\right\|_{\infty} \leq \gamma_{i}
$$

where $\varepsilon_{c, i}$ is defined in (35) for $i=1, \ldots, M-2$ and in (37) for $i=0$ and $i=M-1$.

Theorem 3. Given the transmitters $F_{i}, i=0,1, \ldots, M-1$, there exist receivers $H_{i}(z), i=0,1 \ldots, M-1$, that achieve desirable signal-to-crosstalk ratio (SCR) $\rho$ for all bands if and only if the following LMIs are satisfied:

$$
\left[\begin{array}{cccc}
P_{i} & P_{i} \mathcal{A}_{c, i} & P_{i} \mathcal{B}_{c, i} & 0 \\
(*)^{T} & P_{i} & 0 & \mathcal{C}_{c, i}^{T} \\
(*)^{T} & (*)^{T} & I & \mathcal{D}_{c, i}^{T} \\
(*)^{T} & (*)^{T} & (*)^{T} & 10^{-\rho_{i} / 10} I
\end{array}\right]>0
$$

for $i=0,1, \ldots, M-1$, simultaneously, where $\mathcal{A}_{c, i}, \mathscr{B}_{c, i}, \mathcal{C}_{c, i}$, $D_{c, i}$ are the state-space matrices of $\mathcal{E}_{c, i}$ as defined in (36) and (38).

Remark 4. Note again that (41) is linear in receiver parameters and can be solved using convex optimization. With Theorems 1 and 3, the problem of designing receivers that minimize the reconstruction errors while satisfying the crosstalk attenuation constraint can be solved by the convex optimization in (28) subject to the LMI constraint of (29) and (33).

Remark 5. Note that Chen et al. in [17] discussed a mixed $\mathrm{H}_{2} / \mathrm{H}_{\infty}$ design of noisy transmultiplexer system with respect to inputs. Here, we are concerned with the optimal $H_{2}$ reconstruction of inputs subject to constraints on crosstalk attenuation. 
TABLE 1: Reconstruction performance comparison between different receiver design approaches.

\begin{tabular}{|c|c|c|c|c|c|c|}
\hline \multirow{2}{*}{$\begin{array}{c}\text { Constraint } \\
\gamma\end{array}$} & \multirow{2}{*}{$\mathrm{SNR}_{0}^{r}(\mathrm{~dB})$} & \multicolumn{5}{|c|}{$\mathrm{SNR}^{c}(\mathrm{~dB})$} \\
\hline & & 10 & 15 & 20 & 25 & 30 \\
\hline \multirow{2}{*}{$\gamma=1$} & By proposed method & 30.7518 & 35.3302 & 39.2135 & 41.8637 & 43.2044 \\
\hline & By method in [17] & 30.7450 & 35.3100 & 39.1938 & 41.7722 & 43.0802 \\
\hline \multirow{2}{*}{$\gamma=0.1$} & By proposed method & 30.7476 & 35.3177 & 39.1828 & 41.8071 & 43.1274 \\
\hline & By method in [17] & 30.5784 & 34.9161 & 38.3080 & 40.3420 & 41.2496 \\
\hline
\end{tabular}

TABLE 2: TMUX system SNRs and SCRs comparison for different receiver designs.

\begin{tabular}{|c|c|c|c|c|c|c|c|}
\hline & & \multicolumn{3}{|c|}{$\mathrm{SNR}^{c}=10 \mathrm{~dB}$} & \multicolumn{3}{|c|}{$\mathrm{SNR}^{c}=20 \mathrm{~dB}$} \\
\hline & & Band $\# 0$ & Band $\# 1$ & Band $\sharp 2$ & Band $\sharp 0$ & Band $\# 1$ & Band $\sharp 2$ \\
\hline \multirow{2}{*}{ PR approach } & SCR & 30.8980 & 26.1188 & 23.2797 & 30.8980 & 26.1188 & 23.2797 \\
\hline & $\mathrm{SNR}^{r}$ & 6.7252 & 4.7539 & 4.1931 & 11.6430 & 9.4181 & 8.8873 \\
\hline \multirow{2}{*}{$\begin{array}{l}\text { Optimal } H_{2}+H_{\infty} \text { constraint } \\
\quad\left(\rho_{0}=\rho_{1}=\rho_{2}=30 \mathrm{~dB}\right)\end{array}$} & SCR & 31.8695 & 34.1238 & 32.0317 & 33.8104 & 35.6299 & 34.6269 \\
\hline & $\mathrm{SNR}^{r}$ & 7.8125 & 6.2832 & 5.8382 & 12.2238 & 10.5223 & 9.8985 \\
\hline \multirow{2}{*}{$\begin{array}{l}\text { Optimal } H_{2}+H_{\infty} \text { constraint } \\
\left(\rho_{0}=70, \rho_{1}=\rho_{2}=40 \mathrm{~dB}\right)\end{array}$} & SCR & 74.7892 & 45.9977 & 48.9783 & 76.1100 & 54.5022 & 48.627 \\
\hline & $\mathrm{SNR}^{r}$ & 6.1514 & 6.0711 & 5.5448 & 6.6835 & 10.1444 & 8.5923 \\
\hline
\end{tabular}

\section{EXAMPLES}

\subsection{Example 1}

Now we address the TMUX reconstruction problem. The model presented in [17] is considered, and we design the receivers by our proposed mixed $H_{2} / H_{\infty}$ approach. Firstly, we define the measurement metrics on channel noise (channel signal-to-noise ratio, $\mathrm{SNR}^{c}$ ) and reconstruction performance (reconstruction SNR on the $i$ th band, $\mathrm{SNR}_{i}^{r}$ ) as

$$
\begin{gathered}
\mathrm{SNR}^{c}=10 \log _{10} \frac{\sum_{k=0}^{\infty} p^{2}(k)}{\sum_{k=0}^{\infty} r^{2}(k)}, \\
\mathrm{SNR}_{i}^{r}=10 \log _{10} \frac{\sum_{k=0}^{\infty} s_{i}^{2}(k)}{\sum_{k=0}^{\infty}\left(\hat{s}_{i}(k)-s_{i}^{d}(k)\right)^{2}} .
\end{gathered}
$$

Then the results (on the first band) are listed in Table 1. From it, it is clear that our proposed approach has a slightly better reconstruction performances than the conservative method presented in [17], because of adopting different Lyapunov matrices for the $\mathrm{H}_{2}$ and $\mathrm{H}_{\infty}$ performances. Moreover, the more constraint on $H_{\infty}$ performance is added, the more obvious improvement will produce.

\subsection{Example 2}

In this example, we will examine the crosstalk attenuation performance of a TMUX system. We consider a 3-channel filter bank model in [22], where a perfect reconstruction filter bank has been designed. We adopt its dual system for a 3-band PR TMUX system model.

Under the channel noise of variance $\sigma_{r}^{2}=0.09$ and $\sigma_{r}^{2}=0.9$ (in this case, corresponding to the $\mathrm{SNR}^{c}$ of $20 \mathrm{~dB}$ and $10 \mathrm{~dB}$, resp.), we design the receivers by the optimal $H_{2}$ design (Theorem 1 ) with an $H_{\infty}$ crosstalk constraint
(Theorem 3). A comparison is made with the original perfect reconstruction (PR) TMUX system in Table 2, under different constraints SCRs as defined in (39).

From this table, it can be seen that, firstly, the PR design is inferior to the proposed optimal design with an $H_{\infty}$ crosstalk constraint in both the reconstruction performance and the crosstalk attenuation; secondly, our proposed $H_{\infty}$ constraint can obtain any desired crosstalk attenuation requirement; thirdly, when a stringent crosstalk attenuation is required, the reconstruction performance could be very poor, which shows that in some noisy TMUX system design, a trade-off between crosstalk attenuation and reconstruction performances is to be made.

It is worth pointing out that the overall reconstruction performance is not very good for the example mainly due to the significant frequency overlapping of the three transmitters.

\section{CONCLUSION}

In this paper, we have investigated the optimal receivers design for noisy transmultiplexer systems with the goal of optimizing the reconstruction error while ensuring the crosstalk attenuation below a given level. The former is optimized by $\mathrm{H}_{2}$ approach, while the latter is formulated and solved by $H_{\infty}$ approach. The simulation results indicated that in noisy situations, the proposed design improves the system performance in both the reconstruction and crosstalk attenuation, when compared with the biorthogonal transmultiplexer design approach.

\section{REFERENCES}

[1] M. Bellanger and J. L. Daguet, "TDM-FDM transmultiplexer: digital polyphase and FFT," IEEE Transactions on Communications, vol. 22, no. 9, pp. 1199-1205, 1974. 
[2] Y.-P. Lin and S.-M. Phoong, "ISI-free FIR filterbank transceivers for frequency-selective channels," IEEE Transactions on Signal Processing, vol. 49, no. 11, pp. 2648-2658, 2001.

[3] M. Vetterli, "A theory of multirate filter banks," IEEE Transactions on Acoustics, Speech, and Signal Processing, vol. 35, no. 3, pp. 356-372, 1987.

[4] H. Scheuermann and H. Göckler, "A comprehensive survey of digital transmultiplexing methods," Proceedings of the IEEE, vol. 69, no. 11, pp. 1419-1450, 1981.

[5] P. P. Vaidyanathan, Multirate Systems and Filter Banks, Prentice-Hall, Englewood Cliffs, NJ, USA, 1993.

[6] M. Vetterli, "Perfect transmultiplexers," in Proceedings of IEEE International Conference on Acoustics, Speech, and Signal Processing (ICASSP '86), vol. 11, pp. 2567-2570, Tokyo, Japan, April 1986.

[7] R. D. Koilpillai, T. Q. Nguyen, and P. P. Vaidyanathan, "Some results in the theory of crosstalk-free transmultiplexers," IEEE Transactions on Signal Processing, vol. 39, no. 10, pp. 21742183, 1991.

[8] J. Critchley and P. J. W. Rayner, "Design methods for periodically time varying digital filters," IEEE Transactions on Acoustics, Speech, and Signal Processing, vol. 36, no. 5, pp. 661-673, 1988.

[9] J. S. Prater and C. M. Loeffler, "Analysis and design of periodically time-varying IIR filters, with applications to transmultiplexing," IEEE Transactions on Signal Processing, vol. 40, no. 11, pp. 2715-2725, 1992.

[10] T. Liu and T. Chen, " $H_{2}$ optimization applied to general transmultiplexer design," in Proceedings of the 39th IEEE Conference on Decision and Control, vol. 5, pp. 4314-4319, Sydney, NSW, Australia, December 2000.

[11] Y.-P. Lin and S.-M. Phoong, "Perfect discrete multitone modulation with optimal transceivers," IEEE Transactions on Signal Processing, vol. 48, no. 6, pp. 1702-1711, 2000.

[12] B.-S. Chen and L.-M. Chen, "Optimal reconstruction in multirate transmultiplexer systems under channel noise: Wiener separation filtering approach," Signal Processing, vol. 80, no. 4, pp. 637-657, 2000.

[13] B.-S. Chen, C.-W. Lin, and Y.-L. Chen, "Optimal signal reconstruction in noisy filter bank systems: multirate Kalman synthesis filtering approach," IEEE Transactions on Signal Processing, vol. 43, no. 11, pp. 2496-2504, 1995.

[14] B.-S. Chen and C.-W. Lin, "Optimal design of deconvolution filters for stochastic multirate signal systems," Signal Processing, vol. 47, no. 3, pp. 287-305, 1995.

[15] C.-W. Lin and B.-S. Chen, "State space model and noise filtering design in transmultiplexer systems," Signal Processing, vol. 43, no. 1, pp. 65-78, 1995.

[16] Y.-M. Cheng, B.-S. Chen, and L.-M. Chen, "Minimax deconvolution design of multirate systems with channel noises: a unified approach," IEEE Transactions on Signal Processing, vol. 47, no. 11, pp. 3145-3149, 1999.

[17] B.-S. Chen, C.-L. Tsai, and Y.-F. Chen, "Mixed $H_{2} / H_{\infty}$ filtering design in multirate transmultiplexer systems: LMI approach," IEEE Transactions on Signal Processing, vol. 49, no. 11, pp. 2693-2701, 2001.

[18] P. Gahinet, A. Nemirovski, A. J. Laub, and M. Chilali, LMI Control Toolbox-for Use with MATLAB, The MathWorks, Natick, Mass, USA, 1995.
[19] N. Al-Dhahir and J. M. Cioffi, "Optimum finite-length equalization for multicarrier transceivers," IEEE Transactions on Communications, vol. 44, no. 1, pp. 56-64, 1996.

[20] S. Boyd, L. El Ghaoui, E. Feron, and V. Balakrishnan, Linear Matrix Inequalities in System and Control Theory, SIAM, Philadelphia, Pa, USA, 1994.

[21] L. C. Chu and M. Brooke, "A study on multiuser DSL channel capacity with crosstalk environment," in Proceedings of the IEEE Pacific Rim Conference on Communications, Computers and signal Processing (PACRIM '01), vol. 1, pp. 176-179, Victoria, BC, Canada, August 2001.

[22] C. Zhang and Y. Liao, "A sequentially operated periodic FIR filter for perfect reconstruction," Circuits, Systems, and Signal Processing, vol. 16, no. 4, pp. 475-486, 1997.

Huan Zhou received the B.E. and M.E. degrees in information engineering from Northeastern University in 1994 and 1999, respectively, and the Ph.D. degree in electrical and electronic engineering from the Nanyang Technological University, Singapore, in 2003. She worked as a Postdoctoral Fellow in International Graduate School for Neurosensory Science and Systems, Germany, in 2004. Currently, she is working with Panasonic Singapore Laboratories, focused on AV systems' research and development.

Lihua Xie received the B.E. and M.E. degrees in electrical engineering from Nanjing University of Science and Technology in 1983 and 1986, respectively, and the Ph.D. degree in electrical engineering from the University of Newcastle, Australia, in 1992. He is currently a Professor with the School of Electrical and Electronic Engineering, Nanyang Technological University, Singapore. He held teaching appointments in the

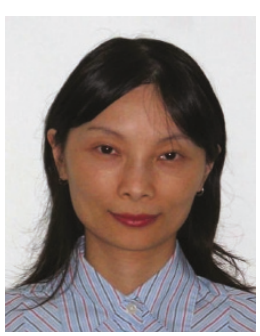
Department of Automatic Control, Nanjing University of Science and Technology from 1986 to 1989 . He also held visiting appointments with the University of Melbourne and the Hong Kong Polytechnic University. His current research interests include estimation theory, robust control, networked control systems, and time delay systems. In these areas, he has published many papers and coauthored (with C. Du) the monograph $\mathrm{H}$-infinity Control and Filtering of Two-dimensional Systems (Springer, 2002). He is currently an Associate Editor of the IEEE Transactions on Automatic Control, International Journal of Control, Automation and Systems, and Journal of Control Theory and Applications. He is also a Member of the Editorial Board of IEE Proceedings on Control Theory and Applications. He served as an Associate Editor of the Conference Editorial Board, IEEE Control Systems Society from 2000 to 2004. 